\title{
ON FELLER'S CRITERION FOR THE LAW OF THE ITERATED LOGARITHM
}

\author{
DELI LI \\ Institute of Mathematics \\ Jilin University \\ Changchun 130023 \\ China \\ and \\ Department of Mathematics \& Statistics \\ University of Regina \\ Regina, Saskatchewan \\ Canada, S4S OA2

\section{BHASKARA RAO} \\ Department of statistics \\ North Dakota State University \\ Fargo, ND 58105
}

USA

\section{XIANGCHEN WANG}

Department of Mathematics

Jilin University

Changchun 130023

China

ABSTRACT. Combining Feller's criterion with a non-uniform estimate result in the context of the Central Limit Theorem for partial sums of independent random variables, we obtain several results on the Law of the Iterated Logarithm. Two of these results refine corresponding results of Wittmann (1985) and Egorov (1971). In addition, these results are compared with the corresponding results of Teicher (1974), Tomkins (1983) and Tomkins (1990)

KEY WORDS AND PHRASES. Central Limit Theorem, Feller's Criterion, Law of the Iterated Logarithm, Non-uniform estimates in the Central Limit Theorem.

1991 AMS SUBJECT CLASSIFICATION CODE. 60F15, 60 F05. 
1. INTRODUCTION

Let $x_{n}, n \geq 1$ be a sequence of independent random variables with zero means and finite variances. Let, for each $n \geq 1$,

$$
\begin{aligned}
& S_{n}=\sum_{i=1}^{n} x_{i}, \quad s_{n}^{2}=\sum_{i=1}^{n} \operatorname{Ex}_{i}^{2}, \quad F_{n}(x)=P\left(S_{n} / s_{n} \leq x\right), \\
& \text { and } \Phi(x)=\int_{-\infty}^{x}(1 / \sqrt{2 \pi}) \exp \left(-t^{2} / 2\right) d t \text { for }-\infty<x<\infty .
\end{aligned}
$$

Feller (1970) proved the following remarkable criterion on the Law of the I terated Logari thm.

THEOREM A. Let $x_{n}, n \geq 1$ be a sequence of independent random variables with zero means and finite variances, and $a_{n}, n \geq 1$ a sequence of positive numbers such that $a_{n} / s_{n} \uparrow \infty$. If there exists a $\rho \geq 0$ such that

$$
\begin{array}{r}
\sum_{n \geq 1} \min \left(1,\left(a_{n+1}-a_{n}\right) / a_{n}\right\} P\left(S_{n}>a_{n} x\right)<\infty \text { for } x>\rho, \\
=\infty \text { for } x<\rho,
\end{array}
$$

then

$$
\limsup _{n \rightarrow \infty} S_{n} / a_{n}=\rho \text { a.s. }
$$

Let $G$ be the collection of all real valued functions $g$ of a real variable with the following properties.

(a) $g$ is nonnegative, even and nondecreasing in the interval $(0, \infty)$.

(b) $x / g(x)$ is nondecreasing in the interval $(0, \infty)$.

Combining Feller's criterion with a non-uniform estimate result in the context of the Central Limit Theorem for partial sums of independent random variables, we obtain several results on the asymptotic behavior of $S_{n} / a_{n}$ with $a_{n} \uparrow \infty$ (Theorems $2.2,2.3,2.4$ and 2.5). We would like to discuss some results of this genre available in the literature and compare them with the results we have derived in this paper. Theorem 1.1 of Wittmann (1985), a certain result of Egorov (1971), Theorem 1 of Teicher (1974), Theorem 1.1 of Tomkins (1983) and Theorem 1 of Tomkins (1990) are some of the results we seek out for comparison. Theorems 2.2 and 2.3 improve the general Law of the Iterated Logarithm due to Wittmann (1985) from which many classical results follow. Theorem 2.4 deals with the case of random variables $x_{n}, n \geq 1$ 
stochastically dominated by a nonnegative random variable $\mathrm{X}$ and improves the corresponding theorem due to Egorov (1971) by weakening the condition from $\mathrm{EX}^{2}<\infty$ to $E\left(\mathrm{X}^{2} / \mathrm{L}_{2} \mathrm{X}\right)<\infty$. ( Here, and in what follows, Lx stands for log max $(x, e)$ and $L_{n} x$ stands for $L\left(L_{n-1} x\right)$ for $n \geq 2$.) In the same vein, two examples are given to compare Theorem 2.5 with Egorov's theorem. Example 4 shows that the conditions in Theorem 2.5 are, in some sense, optimal. Recently, Tomkins (1990) obtained a general result on the Law of the Iterated Logarithm. In Tomkins's paper, no assumptions have been made about the existence of any moments of $x_{n}, n \geq 1$. Our condition (2.3) is similar to Tomkins's condition (5) or (5)' However, our conditions are more easily verifiable. Later, we provide an example satisfying the conditions of Theorem 2.2 and thus of Theorem 2.3 but not those of Theorem 1 of Teicher (1974) and those of Theorem 1.1 of Tomkins (1983). See Example 1 below. He will also provide another example which satisfies the conditions of Theorem 1 of Teicher (1974) as well as the conditions of Theorem 1.1 of Tomkins (1983) but not those of Theorem 2.2. Some additional comments will be made as and when the occasion arises.

It should be remarked that in establishing Laws of Iterated Logarithms, our improvements are achieved by combining the results on non-uniform estimates in the Central Limit Theorem with Theorem A. The arguments appear to be simple.

\section{MAIN RESULTS}

The following result deals with non-uniformestimates in the Central Limit Theorem for sums of independent random variables. A proof can be given following ideas in Katz (1963) and Bikelis (1966).

PROPOSITION 2.1. Let $g \in G$. Let $x_{1}, x_{2}, \cdots, x_{n}$ be independent random variables such that ${E X_{i}}_{i}=0$ and $E\left(X_{i}^{2} g\left(X_{i}\right)\right)<\infty$ for every $i$. Then for every $x$,

$$
\left|F_{n}(x)-\Phi(x)\right| \leq A(1+|x|)^{-2}\left(s_{n}^{2} g\left((1+|x|) s_{n}\right)\right)^{-1} \sum_{i=1}^{n} E\left(X_{i}^{2} g\left(X_{i}\right)\right),
$$

where $A>0$ is an absolute constant. 

l terated logarithm.

THEOREM B. Let $X_{n}, n \geq 1$ be a sequence of independent zero mean real random variables with $\operatorname{Ex}_{n}^{2}<\infty$ for every $n \geq 1$ and $s_{n}^{2}=\sum_{i=1}^{n} \operatorname{Ex}_{i}^{2}, \quad n \geq 1$. If, for some $0<\alpha \leq 1$,

$$
\sum_{n \geq 1}\left(s_{n}^{2} L_{2} s_{n}^{2}\right)^{-(2+\alpha) / 2} E\left|X_{n}\right|^{2+\alpha}<\infty
$$

and

$$
\lim _{n \rightarrow \infty} s_{n}=\infty, \quad \lim \sup _{n \rightarrow \infty}\left(s_{n+1} / s_{n}\right)<\infty,
$$

then

$$
\lim \sup _{n \rightarrow \infty} S_{n} /\left[s_{n}\left(2 L_{2} s_{n}^{2}\right)^{1 / 2}\right]=1 \text { a.s. , }
$$

and

$$
\lim \inf _{n \rightarrow \infty} S_{n} /\left[s_{n}\left(2 L_{2} s_{n}^{2}\right)^{1 / 2}\right]=-1 \text { a.s. }
$$

In the proof of the above theorem, Wittmann (1985) used the direct estimates in the central limit theorem due to Butzer and Hahn (1978). Wittmann (1987) generalized Theorem B covering every $\alpha>0$. Combining Proposition 2.1 and Theorem A, we will generalize Theorem B in a different direction. Our proof seems to be simpler than that given by Wittmann (1985). We would like to emphasize that the generalization of Theorem B by Wittmann (1987) does not imply our generalization of Theorem B.

THEOREM 2.2. Let $X_{n}, n \geq 1$ be a sequence of independent zero mean real random variables with $\mathrm{Ex}_{n}^{2}<\infty$ for every $n \geq 1 \cdot$ and $s_{n}^{2}=\sum_{i=1}^{n} E x_{i}^{2}, n \geq 1$. If (2.2) holds and

$$
\sum_{n \geq 1} E\left(X_{n}^{2} g\left(X_{n}\right)\right) /\left[a_{n}^{2} g\left(a_{n}\right)\right]<\infty \text { for some } g \in G
$$

where $a_{n}=s_{n}\left(L_{2} s_{n}^{2}\right)^{1 / 2}$, then

$$
\lim \sup _{n \rightarrow \infty} S_{n} /\left[s_{n}\left(2 L_{2} s_{n}^{2}\right)^{1 / 2}\right]=1 \text { a.s., }
$$

and

$$
\lim \inf _{n \rightarrow \infty} s_{n} /\left[s_{n}\left(2 L_{2} s_{n}^{2}\right)^{1 / 2}\right]=-1 \text { a.s. }
$$


PROOF. Using Proposition 2.1 and (2.2), we have that for $|x| \geq 1$,

$$
\begin{gathered}
\sum_{n \geq 1} \min \left\{1,\left(a_{n+1}-a_{n}\right) / a_{n}\right\}\left|P\left(S_{n}<x_{n}\left(L_{2} s_{n}^{2}\right)^{1 / 2}\right)-\Phi\left(x_{2}\left(L_{n} s_{n}^{2}\right)^{1 / 2}\right)\right| \\
\leq C_{1} \sum_{n \geq 1}\left[\left(s_{n+1}^{2}-s_{n}^{2}\right) / s_{n}^{2}\right] \sum_{1=1}^{n} E\left(X_{i}^{2} g\left(X_{i}\right)\right) /\left[a_{n}^{2} g\left(a_{n}\right)\right] \\
\leq C_{1} \sum_{i \geq 1}\left(\sum_{n \geq i}\left[s_{n+1}^{2}-s_{n}^{2}\right] /\left[s_{n}^{4} L_{2} s_{n}^{2} g\left(a_{n}\right)\right]\right) E\left(X_{i}^{2} g\left(X_{i}\right)\right) \\
\leq C_{1} \sum_{i \geq 1}\left(\sum_{n \geq i}\left[s_{n+1}^{2}-s_{n}^{2}\right] / s_{n}^{4}\right) E\left(X_{i}^{2} g\left(X_{i}\right)\right) /\left[\left(L_{2} s_{i}^{2}\right) g\left(a_{i}\right)\right] \\
\leq C_{2} \sum_{i \geq 1}\left(\int_{s_{i}^{2}}^{\infty} d x / x^{2}\right) E\left(X_{i}^{2} g\left(X_{i}\right)\right) /\left[\left(L_{2} s_{i}^{2}\right) g\left(a_{i}\right)\right] \\
=C_{2} \sum_{i \geq 1} E\left(X_{i}^{2} g\left(X_{i}\right)\right) /\left[a_{i}^{2} g\left(a_{i}\right)\right]<\infty, \text { by }(2.3)
\end{gathered}
$$

Consequently, if $x \geq 1$, then

$$
\sum_{n \geq 1} \min \left\{1,\left(a_{n+1}-a_{n}\right) / a_{n}\right\} P\left(S_{n}>a_{n} x\right)<\infty
$$

if and only if

$$
\sum_{n \geq 1} \min \left\{1,\left(a_{n+1}-a_{n}\right) / a_{n}\right\}\left(1-\Phi\left(x\left(L_{2} s_{n}^{2}\right)^{1 / 2}\right)<\infty\right.
$$

From (2.2), it is easy to see that (2.5) is equivalent to

$$
\sum_{n \geq 1}\left[\left(s_{n+1}^{2}-s_{n}^{2}\right) / s_{n}^{2}\right]\left(1-\Phi\left(x\left(L_{2} s_{n}^{2}\right)^{1 / 2}\right)<\infty .\right.
$$

Observe that as $n \rightarrow \infty$

$$
\begin{aligned}
& 1-\Phi\left(x\left(L_{2} s_{n}^{2}\right)^{1 / 2}\right) \sim(2 \pi)^{-1 / 2} \exp \left\{-x^{2} L_{2} s_{n}^{2} / 2\right\}\left(x\left(L_{2} s_{n}^{2}\right)^{1 / 2}\right)^{-1} \\
&=(2 \pi)^{-1 / 2}\left(L s_{n}^{2}\right)^{-x^{2} / 2}\left(x\left(L_{2} s_{n}^{2}\right)^{1 / 2}\right)^{-1}
\end{aligned}
$$

Thus for $x \geq 1,(2.6)$ is equivalent to

$$
\sum_{n \geq 1}\left[\left(s_{n+1}^{2}-s_{n}^{2}\right) / s_{n}^{2}\right]\left(L s_{n}^{2}\right)^{-x^{2} / 2}\left(L_{2} s_{n}^{2}\right)^{-1 / 2}<\infty .
$$

Note that

$$
\begin{aligned}
\int_{1}^{\infty} t^{-1}(L t)^{-x^{2} / 2}\left(L_{2} t\right)^{-1 / 2} d t & <\infty \text { for } x>\sqrt{2}, \\
& =\infty \text { for } x \leq \sqrt{2}
\end{aligned}
$$

implies 


$$
\begin{aligned}
\sum_{n \geq 1}\left[\left(s_{n+1}^{2}-s_{n}^{2}\right) / s_{n}^{2}\right]\left(L s_{n}^{2}\right)^{-x^{2} / 2}\left(L_{2} s_{n}^{2}\right)^{-1 / 2} & <\infty \text { for } x>\sqrt{2} \\
& =\infty \text { for } x \leq \sqrt{2} .
\end{aligned}
$$

Therefore

$$
\begin{aligned}
\sum_{n \geq 1} \min \left\{1,\left(a_{n+1}-a_{n}\right) / a_{n}\right\} P\left(S_{n}>a_{n} x\right) & <\infty \text { for } x>\sqrt{2} \\
& =\infty \text { for } x \leq \sqrt{2} .
\end{aligned}
$$

By Theorem A,

$$
\lim \sup _{n \rightarrow \infty} S_{n} /\left[s_{n}\left(2 L_{2} s_{n}^{2}\right)^{1 / 2}\right]=1 \text { a.s. }
$$

and

$$
\lim \inf _{n \rightarrow \infty} s_{n} /\left[s_{n}\left(2 L_{2} s_{n}^{2}\right)^{1 / 2}\right]=-1 \text { a.s. }
$$

by replacing $x_{i}$ by $-X_{i}$.

Looking at the proof of Theorem 2.2, in fact, we have virtually established the following result. The reason that Theorem 2.2 is recast as Theorem 2.3 is that it becomes transperent that the conditions of Theorem 2.3 are weaker than the conditions of Theorem 3 of Petrov (1975), p.305 and the same conclusion holds.

THEOREM 2.3. Let $X_{n}, n \geq 1$ be a sequence of independent zero mean real random variables with $\operatorname{EX}_{n}^{2}<\infty$ for every $n \geq 1$ and $s_{n}^{2}=\sum_{i=1}^{n} E x_{i}^{2}, n \geq 1$. If $s_{n} \rightarrow \infty$ as $n \rightarrow \infty, \quad \limsup _{n \rightarrow \infty} s_{n+1} / s_{n}<\infty$, and for all $x$ such that $|x| \geq 1$,

$$
\sum_{n \geq 1}\left[\left(s_{n+1}^{2}-s_{n}^{2}\right) / s_{n}^{2}\right]\left|P\left(S_{n} / s_{n}<x\left(L_{2} s_{n}^{2}\right)^{1 / 2}\right)-\Phi\left(x\left(L_{2} s_{n}^{2}\right)^{1 / 2}\right)\right|<\infty,
$$

then

$$
\limsup { }_{n \rightarrow \infty} S_{n} /\left[s_{n}\left(2 L_{2} s_{n}^{2}\right)^{1 / 2}\right]=1 \text { a.s. }
$$

and

$$
\liminf _{n \rightarrow \infty} S_{n} /\left[s_{n}\left(2 L_{2} s_{n}^{2}\right)^{1 / 2}\right]=-1 \text { a.s. }
$$

REMARKS. In order to show that Theorem B of Wittmann (1985) is a special case of Theorem 2.2 above, one merely needs to take the function $g$ in Theorem 2.2 to be: $g(x)=|x|^{\alpha}, x \in \mathbb{R}$. One should also note that with 
respect to Theorem 2.2 that Condition (2.3) requires more than finite second moments of the random variables in order for it to hold.

We now give an example for which the conditions of Theorem 2.2 (thus of Theorem 2.3) are satisfied, but neither the conditions of Theorem 1 of Teicher (1974) nor the conditions of Theorem 1.1 of Tomkins (1983) are satisfied.

EXAMPLE 1. Define a sequence $X_{n}, n \geq 1$ of independent random variables as follows: for $n=1,2,3$,

$$
P\left(X_{n}= \pm 1\right) \quad=1 / 2,
$$

and for $n \geq 4$,

$$
\begin{array}{ll}
P\left(X_{n}= \pm 1\right) & =1 / 2-(2 L n)^{-1} ; \\
P\left(X_{n}= \pm n^{1 / 2}\right) & =(2 n L n)^{-1} ; \\
P\left(X_{n}=0\right) & =(L n)^{-1}-(n L n)^{-1}
\end{array}
$$

Clearly, $\operatorname{EX}_{n}=0$ and $\operatorname{EX}_{n}^{2}=1$ for all $n$. Consequently, $s_{n}^{2}=n$ for all $n$. Note that, for $n \geq 4$,

$$
E\left|X_{n}\right|^{3}=1-(L n)^{-1}+n^{1 / 2}(L n)^{-1},
$$

and thus,

$$
\sum_{n \geq 1}\left(n L_{2} n\right)^{-3 / 2} E\left|x_{n}\right|^{3}<\infty .
$$

Therefore, by Theorem 2.2,

$$
\limsup _{n \rightarrow \infty} S_{n} /\left(2 n L_{2} n\right)^{1 / 2}=1 \text { a.s., }
$$

and

$$
\liminf _{n \rightarrow \infty} S_{n} /\left(2 n L_{2} n\right)^{1 / 2}=-1 \text { a.s. }
$$

However, since

$$
\sum_{n \geq 3}(n L n)^{-1}=\infty,
$$

and

$$
\sum_{n \geq 3}\left(\mathrm{~nL}_{3} \mathrm{n}\right)^{-1}=\infty \text {, }
$$

it can be verified that for every $\varepsilon>0$ and $\delta>0$,

$$
\sum_{n \geq 1} P\left(\left|X_{n}\right|>\varepsilon n^{1 / 2}\left(L_{2} n\right)^{-1 / 2}\right)=\infty
$$

and

$$
\sum_{n \geq 1}\left(n L_{2} n\right)^{-1} E\left(X_{n}^{2} I\left(\varepsilon n^{1 / 2}\left(L_{2} n\right)^{-1 / 2}<\left|X_{n}\right| \leq \delta\left(n L_{2} n\right)^{1 / 2}\right)\right)=\infty
$$


Thus Theorem 1 of Teicher (1974) and Theorem 1.1 of Tomkıns (1983) are not applicable here.

The next example satisfies the conditions of Kolmogorov's Law of the Iterated Logarithm (thus of Theorem 1 of Teicher (1974) and of Theorem 1.1 of Tomkins (1983)), but not those of Theorem 2.2 above.

EXAMPLE 2. Let $x_{n}, n \geq 1$ be a sequence of independent random variables such that for each $n \geq 1$,

$$
P\left(X_{n}=0\right)=1-n^{-1}\left(L_{2} n\right)\left(L_{3} n\right) \text {, }
$$

and

$$
P\left(X_{n}= \pm n^{1 / 2}\left(L_{2} n\right)^{-1 / 2}\left(L_{3} n\right)^{-1 / 2}\right)=(2 n)^{-1}\left(L_{2} n\right)\left(L_{3} n\right) .
$$

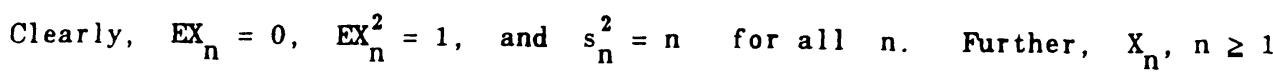
satisfies the conditions of Kolmogorov's Law of the Iterated Logarithm. Thus we have

$$
\limsup _{n \rightarrow \infty} S_{n} /\left(2 n L_{2} n\right)^{1 / 2}=1 \text { a.s. }
$$

and

$$
\liminf _{n \rightarrow \infty} S_{n} /\left(2 n L_{2} n\right)^{1 / 2}=-1 \text { a.s. }
$$

However, for any $g \in G$,

$$
\begin{aligned}
& \sum_{n \geq 1} E\left[X_{n}^{2} g\left(X_{n}\right)\right] /\left[n L_{2} n g\left(\left(n L_{2} n\right)^{1 / 2}\right)\right] \\
& =\sum_{n \geq 1}\left[n\left(L_{2} n\right)^{-1}\left(L_{3} n\right)^{-1} g\left(\left(n /\left(\left(L_{2} n\right)\left(L_{3} n\right)\right)\right)^{1 / 2}\right)\right] /\left[n L_{2} n g\left(\left(n L_{2} n\right)^{1 / 2}\right)\right] \\
& \geq \sum_{n \geq 1} n^{-1}\left(L_{2} n\right)^{-2}\left(L_{3} n\right)^{-1 / 2}=\infty \quad(\text { since } x / g(x) \uparrow) .
\end{aligned}
$$

Thus Theorem 2.2 is not applicable here.

Al though Kolmogorov's Law of the Iterated Logarithm (thus Theorem 1 of Teicher (1974) and Theorem 1.1 of Tomkins (1983)) does not follow from Theorem 2.2 above, we conjecture that Theorem 1 of Teicher (1974) and Theorem 1.1 of Tomkins (1983) (thus Kolmogorov's Law of the Iterated Logarithm) follow from Theorem 2.3 above. In fact, the classical Hartman-Wintner Law of the Iterated Logarithm (1941) follows from our work. Let $X, X_{n}, n \geq 1$ be a sequence of independent identically distributed random variables such that $\mathrm{EX}=0$ and $\mathrm{EX}^{2}=1$. Set

$$
\sigma_{n}^{2}=E\left(X^{2} I\left(|X|<n^{1 / 2}\right)\right)-\left(E\left(X I\left(|X|<n^{1 / 2}\right)\right)\right)^{2}, n \geq 1 .
$$


By Friedman-Katz-Koopmans Theorem (1966),

$$
\sum_{n \geq 1} n^{-1} \sup _{x}\left|P\left(\sigma_{n}^{-1} n^{-1 / 2} \sum_{j=1}^{n} x_{j}<x\right)-\Phi(x)\right|<\infty .
$$

Note that $\sigma_{n} \rightarrow 1$ as $n \rightarrow \infty$. By Theorem 2.3, it follows that

$$
\limsup _{n \rightarrow \infty} S_{n} /\left(2 n L_{2} n\right)^{1 / 2}=1 \text { a.s. }
$$

and

$$
\liminf _{n \rightarrow \infty} S_{n} /\left(2 n L_{2} n\right)^{1 / 2}=-1 \text { a.s. }
$$

Let $X_{n}, n \geq 1$ be a sequence of independent zero mean random variables, $g \in G$, and $a_{n}, n \geq 1$ a sequence of positive numbers such that $a_{n} \uparrow \infty$ as $n \rightarrow \infty$. Using Theorem 9 of Petrov (1975), p.267 and Kronecker's lemma, one can show that

$$
\sum_{n \geq 1} E\left[\left|x_{n}\right| g\left(x_{n}\right)\right] /\left[a_{n} g\left(a_{n}\right)\right]<\infty
$$

implies

$$
S_{n} / a_{n} \rightarrow 0 \text { a.s. as } n \rightarrow \infty \text {. }
$$

Theorem 2.2 shows that if (2.2) holds and $a_{n}=s_{n}\left(L_{2} s_{n}^{2}\right)^{1 / 2}, n \geq 1$, then

$$
\sum_{n \geq 1} E\left[\left|x_{n}\right|^{2} g\left(x_{n}\right)\right] /\left[a_{n}^{2} g\left(a_{n}\right)\right]<\infty
$$

implies that $x_{n}, n \geq 1$ obeys the Law of the Iterated Logarithm. Note that conditions (2.7) and (2.9) do not imply each other. We now give a result whose conclusion is in the spirit of (2.8) under a condition which is in the spirit of (2.9). This result is a consequence of Proposition 2.1 and Theorem A.

THEOREM 2.4. Let $X_{n}, n \geq 1$ be a sequence of independent zero mean random variables with $\operatorname{Ex}_{n}^{2}<\infty$ for every $n \geq 1$ and $s_{n}^{2}=\sum_{i=1}^{n} \operatorname{Ex}_{i}^{2}, n \geq 1$. Let $a_{n}$, $n \geq 1$ be a sequence of positive numbers and $g \in G$. Suppose that

$$
\begin{gathered}
a_{n} / s_{n} \uparrow, \quad \limsup _{n \rightarrow \infty} a_{n+1} / a_{n}<\infty, \\
\lim { }_{n \rightarrow \infty} s_{n}\left(L_{2} s_{n}^{2}\right)^{1 / 2} / a_{n}=0,
\end{gathered}
$$

and

$$
\sum_{n \geq 1}\left[\operatorname{EX}_{n}^{2} g\left(X_{n}\right)\right] /\left[a_{n}^{2} g\left(a_{n}\right)\right]<\infty .
$$

Then

$$
s_{n} / a_{n} \rightarrow 0 \text { a.s. as } n \rightarrow \infty \text {. }
$$


PROOF. It follows from Proposition 2.1 that for every $x \neq 0$

$$
\begin{aligned}
& \left|P\left(S_{n} / s_{n}<x a_{n} / s_{n}\right)-\Phi\left(x a_{n} / s_{n}\right)\right| \\
& \quad \leq A\left[\sum_{i=1}^{n} \operatorname{Ex}_{i}^{2} g\left(X_{i}\right)\right] /\left[a_{n}^{2} g\left(a_{n}\right)\right],
\end{aligned}
$$

where $A>0$ is a constant depending only on $x$. Using (2.10), we have that for $x \neq 0$,

$$
\begin{aligned}
\sum_{n \geq 1} \min (1, & \left.\left(a_{n+1}-a_{n}\right) / a_{n}\right\}\left|P\left(S_{n}<x a_{n}\right)-\Phi\left(x_{n} / s_{n}\right)\right| \\
\leq A & \sum_{n \geq 1}\left[\left(a_{n+1}-a_{n}\right) / a_{n}\right]\left[\sum_{i=1}^{n} \operatorname{Ex}_{i}^{2} g\left(X_{i}\right)\right] /\left[a_{n}^{2} g\left(a_{n}\right)\right] \\
& =A \sum_{i \geq 1}\left\{\sum_{n \geq i}\left(a_{n+1}-a_{n}\right) /\left[a_{n}^{3} g\left(a_{n}\right)\right]\right\} \operatorname{Ex}_{i}^{2} g\left(X_{i}\right) \\
& \leq C \sum_{i \geq 1}\left(\int_{a_{i}}^{\infty}\left[t^{3} g\left(a_{i}\right)\right]^{-1} d t\right) \operatorname{Ex}_{i}^{2} g\left(X_{i}\right) \\
& \leq C \sum_{i \geq 1}\left[\operatorname{Ex}_{i}^{2} g\left(X_{i}\right)\right] /\left[a_{i}^{2} g\left(a_{i}\right)\right]<\infty,
\end{aligned}
$$

where $C$ is also a constant depending only on $x$. Therefore, for every $\epsilon>0$

$$
\sum_{n \geq 1} \min \left\{1,\left(a_{n+1}-a_{n}\right) / a_{n}\right\} P\left(\left|S_{n}\right| \geq \varepsilon a_{n}\right)<\infty
$$

if and only if

$$
\sum_{n \geq 1} \min \left\{1,\left(a_{n+1}-a_{n}\right) / a_{n}\right\} \Phi\left(-\varepsilon a_{n} / s_{n}\right)<\infty .
$$

Observe that as $n \rightarrow \infty$

$$
\Phi\left(-\epsilon a_{n} / s_{n}\right)=0\left(\exp \left\{-\varepsilon^{2} a_{n}^{2} / 2 s_{n}^{2}\right\}\right)
$$

Since $t / L_{2} t$ is monotonically increasing on $(0, \infty)$, then $\left[s_{n}^{2} L_{2} s_{n}^{2}\right] / a_{n}{ }^{2} \rightarrow 0$ as $n \rightarrow \infty$ if and only if $\left[s_{n}^{2} L_{2} s_{n}^{2}\right] /\left[\left(a_{n}^{2} / L_{2} a_{n}^{2}\right) L_{2}\left(a_{n}^{2} / L_{2} a_{n}^{2}\right)\right] \rightarrow 0$ as $n \rightarrow \infty$. Consequently, (2.11) implies $\left[s_{n}^{2} L_{2} a_{n}\right] / a_{n}^{2} \rightarrow 0$, i.e., $s_{n}^{2} / a_{n}^{2}=0\left(1 / L_{2} s_{n}^{2}\right)$ as $n \rightarrow \infty$. It now follows that

$$
\exp \left\{-\epsilon^{2} a_{n}{ }^{2} / 2 s_{n}^{2}\right\}=0\left(\exp \left\{-2 L_{2} a_{n}\right\}\right) \text { as } n \rightarrow \infty
$$

From (2.10),

$$
\begin{aligned}
& \sum_{n \geq 1}\left[\left(a_{n+1}-a_{n}\right) / a_{n}\right] \exp \left\{-2 L_{2} a_{n}\right\} \\
& \leq \sum_{n \geq 1}\left(a_{n+1}-a_{n}\right) /\left[a_{n}\left(L a_{n}\right)^{2}\right] \\
& \quad \leq C_{1} \int_{a_{1}}^{\infty}\left[t(L t)^{2}\right]^{-1} d t<\infty .
\end{aligned}
$$


Now we have that (2.13) holds for every $\varepsilon>0$. It is easy to see that

$$
\left.\sum_{n \geq 1} \min \left\{1,: a_{n+1}-a_{n}\right) / a_{n}\right\} P\left(\left|S_{n}\right| \geq \varepsilon a_{n}\right)<\infty \text { for every } \varepsilon>0 .
$$

From Theorem A,

$$
S_{n} / a_{n} \rightarrow 0 \text { a.s. as } n \rightarrow \infty \text {. }
$$

REMARK. If Condition (2.11) is replaced by

$$
\limsup { }_{n \rightarrow \infty} s_{n}\left(L_{2} s_{n}^{2}\right)^{1 / 2} / a_{n}=\Lambda<\infty
$$

one can show by a similar argument that

$$
\limsup { }_{n \rightarrow \infty}\left|S_{n}\right| / a_{n} \leq \sqrt{2} \Lambda \text { a.s. }
$$

When a sequence $X_{n}, n \geq 1$ is stochastically dominated by a nonnegative random variable $X$, Egorov (1971) gave the following sufficient conditions for $x_{n}, n \geq 1$ to obey the Law of the Iterated Logarithm.

THEOREM $C$. Let $X_{n}, n \geq 1$ be a sequence of independent zero mean random variables with $\operatorname{Ex}_{n}^{2}<\infty$ for all $n \geq 1$ and $s_{n}^{2}=\sum_{i=1}^{n} E x_{i}^{2}, n \geq 1$. Suppose there is a nonnegative random variable $X$ such that for sufficiently large n and $x$

$$
(1 / n) \sum_{i=1}^{n} P\left(\left|x_{i}\right|>x\right) \leq P(X>x)
$$

If

$$
\operatorname{Ex}^{2}<\infty
$$

and

$$
\liminf { }_{n \rightarrow \infty} s_{n}^{2} / n>0
$$

then

$$
\limsup _{n \rightarrow \infty} S_{n} /\left[s_{n}\left(2 L_{2} s_{n}^{2}\right)^{1 / 2}\right]=1 \text { a.s. , }
$$

and

$$
\liminf { }_{n \rightarrow \infty} S_{n} /\left[s_{n}\left(2 L_{2} s_{n}^{2}\right)^{1 / 2}\right]=-1 \text { a.s. }
$$

As a last application of Proposition 2.1, we shall extend the above theorem. 
THEOREM 2.5. Let $X_{n}, n \geq 1$ be a sequence of independent zero mean random varrables with $\operatorname{Ex}_{n}^{2}<\infty$ for all $n \geq 1$ and $s_{n}^{2}=\sum_{i=1}^{n} \operatorname{Ex}_{i}^{2}, n \geq 1$. Suppose there exists a nonnegative random variable $X$ such that for all sufficiently large $n$ and $x$

$$
(1 / n) \sum_{i=1}^{n} P\left(\left|X_{i}\right|>x\right) \leq P(X>x) .
$$

If

$$
E\left(X^{2} / L_{2} X\right)<\infty
$$

and

$$
0<\liminf _{n \rightarrow \infty} s_{n}^{2} / n \leq \limsup _{n \rightarrow \infty} s_{n}^{2} / n<\infty,
$$

then

$$
\limsup { }_{n \rightarrow \infty}\left|S_{n}\right| /\left[s_{n}\left(2 L_{2} s_{n}^{2}\right)^{1 / 2}\right] \leq 1 \text { a.s. }
$$

Moreover, if the following additional condition is satisfied

$$
\lim _{n \rightarrow \infty}(1 / n) \sum_{i=1}^{n} E\left(X_{i}^{2} I\left(\left|X_{i}\right| \geq \sqrt{i L_{2} i}\right)\right)=0
$$

then

$$
\limsup { }_{n \rightarrow \infty} S_{n} /\left[s_{n}\left(2 L_{2} s_{n}^{2}\right)^{1 / 2}\right]=1 \text { a.s. }
$$

and

$$
\liminf _{n \rightarrow \infty} S_{n} /\left[s_{n}\left(2 L_{2} s_{n}^{2}\right)^{1 / 2}\right]=-1 \text { a.s. }
$$

PROOF. For each $n \geq 1$, let

$$
\begin{aligned}
& Y_{n}=X_{n} I\left(\left|X_{n}\right|<\sqrt{n L_{2} n}, \quad Z_{n}=X_{n}-Y_{n},\right. \\
& U_{n}=Y_{n}-E Y_{n}, \quad V_{n}=Z_{n}-E Z_{n}, \quad S_{n}^{\prime 2}=\sum_{i=1}^{n} E U_{n}^{2} .
\end{aligned}
$$

We first prove that from (2.17) and (2.19)

$$
\left(\sum_{i=1}^{n} V_{i}\right) / \sqrt{2 n L_{2} n} \rightarrow 0 \text { a.s. as } n \rightarrow \infty .
$$

Observe that if $N$ is large enough (assume $N=2^{m-1}$ for some $m \geq 1$ )

$$
\begin{aligned}
\sum_{n \geq N} P\left(Z_{n} \neq 0\right) & =\sum_{n \geq N} P\left(\left|x_{n}\right| \geq \sqrt{n L_{2} n}\right) \\
& \leq \sum_{k \geq m} \sum_{n=2}^{2^{k}} P\left(\left|x_{n}\right| \geq \sqrt{n L_{2} n}\right) \\
& \leq \sum_{k \geq m} 2^{k} P\left(X \geq\left(2^{k-1} L_{2} 2^{k-1}\right)^{1 / 2}\right)
\end{aligned}
$$




$$
\begin{aligned}
& \leq 4 \int_{0}^{\infty} \mathrm{P}\left(\mathrm{X}^{2} \geq \mathrm{x} \mathrm{L}_{2} \mathrm{X}\right) \mathrm{dx} \\
& \leq 4 \int_{0}^{\infty} \mathrm{P}\left(\mathrm{X}^{2} / \mathrm{L}_{2} \mathrm{X} \geq(1 / 2) \mathrm{x}\right) \mathrm{dx} \\
& =16 \mathrm{E}\left(\mathrm{X}^{2} / \mathrm{L}_{2} \mathrm{X}\right)<\infty .
\end{aligned}
$$

It follows that

$$
\sum_{i=1}^{n} Z_{i} / \sqrt{2 n L_{2} n} \rightarrow 0 \text { a.s. as } n \rightarrow \infty \text {. }
$$

Note that (2.19) and $\left|\mathrm{EZ}_{n}\right| / \mathrm{Ex}_{n}^{2} \leq\left[\mathrm{Ex}_{n}^{2} / \sqrt{n \mathrm{~L}_{2} \mathrm{n}}\right] / \mathrm{Ex}_{n}^{2} \rightarrow 0$ as $\mathrm{n} \rightarrow \infty$ imply

$$
\sum_{i=1}^{n} E Z_{i} / s_{n}^{2} \rightarrow 0 \text { as } n \rightarrow \infty
$$

Now (2.23), (2.24) and (2.19) show that (2.22) holds which is equivalent to

$$
\sum_{i=1}^{n} V_{i} /\left[s_{n}\left(2 L_{2} s_{n}^{2}\right)^{1 / 2}\right] \rightarrow 0 \text { a.s. as } n \rightarrow \infty
$$

by (2.19). To complete the proof of (2.20), we only need to prove

$$
\sum_{n \geq 1} E\left|U_{n}\right|^{3} /\left(n L_{2} n\right)^{3 / 2}<\infty
$$

in view of (2.19), $\mathrm{s}_{\mathrm{n}}^{\prime} / \mathrm{s}_{\mathrm{n}} \leq 1$ and Remark. If $\mathrm{N}$ is large enough

$$
\begin{aligned}
& \sum_{n \geq N} E\left|U_{n}\right|^{3} /\left(n L_{2} n\right)^{3 / 2} \leq 24 \sum_{n \geq N}\left[\int_{0}^{\sqrt{n L_{2} n}} t^{2} P\left(\left|X_{n}\right| \geq t\right) d t\right] /\left(n L_{2} n\right)^{3 / 2} \\
& \leq 24 \sum_{n \geq N}\left[\int_{0}^{s} t^{2} d t\right] /\left(n L_{2} n\right)^{3 / 2} \\
& +24 \sum_{n \geq N}\left[\int_{s}^{\sqrt{n L_{2} n}} t^{2} P\left(\left|x_{n}\right| \geq t\right) d t\right] /\left(n L_{2} n\right)^{3 / 2}
\end{aligned}
$$

where $s$ is a fixed number large enough. Obviously, the first series converges. By (2.17) and (2.18), we estimate the second series by following

$$
\begin{aligned}
& \sum_{n \geq N}\left[\int_{s}^{\sqrt{n L}{ }^{n}} t^{2} P\left(\left|x_{n}\right| \geq t\right) d t\right] /\left(n L_{2} n\right)^{3 / 2} \\
& \quad \leq \sum_{k \geq m}\left[\int_{s}^{\left(2^{k} L_{2} 2^{k}\right)^{1 / 2}} t^{2} \sum_{i=2^{k-1}}^{2^{k}} P\left(\left|x_{i}\right| \geq t\right) d t\right] /\left(2^{k-1} L_{2} 2^{k-1}\right)^{3 / 2}, \\
& \quad \text { or some suitable m, } \\
& \leq 8 \sum_{k \geq m}\left[\int_{0}^{\left(2^{k} L_{2} 2^{k}\right)^{1 / 2}} t^{2} 2^{k} P(x \geq t) d t\right] /\left(2^{k} L_{2} 2^{k}\right)^{3 / 2}
\end{aligned}
$$




$$
\begin{aligned}
& =8 \sum_{k \geq m} \sum_{i=1}^{k}\left[\int_{b_{i-1}}^{b_{i}} t^{2} 2^{k} P(X \geq t) d t\right]\left(2^{k_{L}} 2^{k}\right)^{3 / 2} \\
& \leq 8 \sum_{k \geq m} \sum_{i=1}^{k}\left[b_{i}^{3} 2^{k} P\left(X \geq b_{i-1}\right)\right] /\left(2^{k_{L}} 2^{k}\right)^{3 / 2} \\
& \leq 8 \sum_{1 \geq 1}\left[\sum_{k \geq 1} 2^{-k / 2}\left(L_{2} 2^{k}\right)^{-3 / 2}\right] b_{i}^{3} P\left(X \geq b_{i-1}\right) \\
& \leq 32 \sum_{i \geq 1} 2^{-i / 2}\left(L_{2} 2^{i}\right)^{-3 / 2} b_{i}^{3} P\left(X \geq b_{i-1}\right) \\
& =64\left[\sum_{i \geq 1} 2^{i-1} P\left(X \geq\left(2^{i-1} L_{2} 2^{i-1}\right)^{1 / 2}\right)+1\right]<\infty .
\end{aligned}
$$

We conclude that $(2.20)$ holds.

Under the conditions (2.19) and (2.21), it is easy to verify that $\lim _{n \rightarrow \infty} s_{n}^{\prime} / s_{n}=1$, and further, it follows from (2.25), (2.26) and Theorem 2.2 that

$$
\limsup _{n \rightarrow \infty} S_{n} /\left[s_{n}\left(2 L_{2} s_{n}^{2}\right)^{1 / 2}\right]=1 \text { a.s. }
$$

and

$$
\liminf { }_{n \rightarrow \infty} s_{n} /\left[s_{n}\left(2 L_{2} s_{n}^{2}\right)^{1 / 2}\right]=-1 \text { a.s. }
$$

In view of the characterization of the law of the iterated logarithm in Banach spaces given in Ledoux and Talagrand (1988), the condition (2.18) merits special interest. Note that the conditions (2.18) and (2.21) both follow from the condition (2.15) under which Theorem C operates. We shall give an example which fails to meet the conditions of Theorem $C$ but Theorem 2.5 is applicable.

EXAMPLE 3. Let $p_{n}=\exp \left\{e^{n^{2}}\right\}, n \geq 1$ and $x_{n}, n \geq 1$ a sequence of independent random variables with distributions given as follows.

$$
\begin{aligned}
P\left(X_{n}=x\right) & =\left(2 p_{n} L_{4} p_{n}\right)^{-1}, \text { if } x= \pm \sqrt{p_{n}} \\
& =(1 / 2)\left(1-1 / L_{4} p_{n}\right), \text { if } x= \pm 1 \\
& =\left(1 / L_{4} p_{n}\right)\left(1-1 / p_{n}\right), \text { if } x=0, \text { for all } n \geq 1
\end{aligned}
$$

It is easy to see that $\operatorname{EX}_{n}=0$ and $\operatorname{Ex}_{n}^{2}=1$ for all $n \geq 1$. Let 


$$
G_{1}(x)=\sup _{n \geq 1}(1 / n) \sum_{1=1}^{n} P\left(\left|x_{i}\right| \geq x\right)
$$

and

$$
G_{2}(x)=\sup _{n \geq 1} P\left(\left|x_{n}\right| \geq x\right), \quad-\infty<x<\infty .
$$

Obviously, $G_{1}(x) \leq G_{2}(x)$ for all $x$ and $G_{2}(x) \rightarrow 0$ as $x \rightarrow \infty$.

Consequently, both $1-G_{1}$ and $1-G_{2}$ are distribution functions. We first show that

$$
\int_{0}^{\infty} x G_{1}(x) d x=\infty
$$

In fact,

$$
\begin{aligned}
\int_{0}^{\infty} x G_{1}(x) d x & \geq \int_{0}^{\infty} x\left[\sup _{n \geq 1}(1 / n) P\left(\left|x_{n}\right| \geq x\right)\right] d x \\
& \geq \sum_{k \geq 1} \int_{\sqrt{p_{k}}}^{\sqrt{p_{k+1}}} \times\left[\sup _{n \geq 1}(1 / n) P\left(\left|x_{n}\right| \geq x\right)\right] d x \\
\geq & \sum_{k \geq 1} \int_{\sqrt{p_{k}}}^{\sqrt{p_{k+1}}}(x /(k+1)) P\left(\left|x_{k+1}\right| \geq x\right) d x \\
& =\sum_{k \geq 1}\left[(k+1) p_{k+1} L_{4} p_{k+1}\right]^{-1} \int_{\sqrt{p_{k}}}^{\sqrt{p_{k+1}}} x d x \\
& \geq(1 / 4) \sum_{k \geq 1} 1 /\left[(k+1) L_{2}(k+1)\right]=\infty .
\end{aligned}
$$

Note that (2.27) implies that (2.15) fails. For, if any nonnegative random variable $Y$ satisfies

$$
(1 / n) \sum_{i=1}^{n} P\left(\left|X_{i}\right| \geq x\right) \leq P(Y \geq x) \text { for all } x \geq 0 \text { and } n \geq 1 \text {, }
$$

then from the definition of $G_{1}(x)$

$$
G_{1}(x) \leq P(Y \geq x) \text { for all } x \geq 0
$$

and from (2.27) we have that $\mathrm{EY}^{2}=\infty$. Now we show that

$$
\int_{0}^{\infty}\left(x / L_{2} x\right) G_{1}(x) d x<\infty
$$

In fact,

$$
\begin{aligned}
\int_{0}^{\infty}\left(x / L_{2} x\right) G_{1}(x) d x \leq & 2 \int_{0}^{\infty}\left(x / L_{2} x^{2}\right) G_{2}(x) d x \\
& =2\left[\int_{0}^{2}\left(x / L_{2} x^{2}\right) G_{2}(x) d x+\int_{2}^{\sqrt{p_{1}}}\left(x / L_{2} x^{2}\right) G_{2}(x) d x\right.
\end{aligned}
$$




$$
\begin{aligned}
& \left.+\sum_{n \geq 1} \int_{\sqrt{p_{n}}}^{\sqrt{p_{n+1}}}\left(x / L_{2} x^{2}\right) G_{2}(x) d x\right] \\
& \leq 2\left[2+p_{1}+\sum_{n \geq 1}\left(p_{n+1} / L_{2} p_{n}\right)\left(1 /\left(p_{n+1} L_{4} p_{n+1}\right)\right]\right. \\
& \leq 2\left[2+p_{1}+\sum_{n \geq 1}\left(1 / n^{2}\right)\right]<\infty .
\end{aligned}
$$

But (2.28) implies that the condition (2.18) holds where $X$ is a random variable with the distribution function $1-G_{1}$. Moreover, as $n \rightarrow \infty$

$$
\operatorname{Ex}_{n}^{2} I\left(\left|X_{n}\right| \geq \sqrt{n L_{2}^{n}}\right)=1 / L_{4} p_{n} \rightarrow 0
$$

It now follows that

$$
\lim _{n \rightarrow \infty}(1 / n) \sum_{i=1}^{n} \operatorname{Ex}_{i}^{2} I\left(\left|X_{i}\right| \geq\left(i L_{2} i\right)^{1 / 2}\right)=0
$$

By Theorem 2.5

$$
\limsup { }_{n \rightarrow \infty} S_{n} / \sqrt{2 n L_{2} n}=1 \text { a.s., }
$$

and

$$
\liminf _{n \rightarrow \infty} S_{n} / \sqrt{2 n L_{2} n}=-1 \text { a.s. }
$$

The following example shows that for a sequence $x_{n}, n \geq 1$ to obey the Law of the Iterated Logarithm, i.e.,

$$
\limsup _{n \rightarrow \infty} S_{n} /\left[s_{n}\left(2 L_{2} s_{n}^{2}\right)^{1 / 2}\right]=1 \text { a.s., }
$$

and

$$
\liminf _{n \rightarrow \infty} s_{n} /\left[s_{n}\left(2 L_{2} s_{n}^{2}\right)^{1 / 2}\right]=-1 \text { a.s. }
$$

in the framework of Theorem 2.5, the condition (2.21) is optimal in a way.

EXAMPLE 4. Let $p_{n}=\exp \left\{e^{n^{2}}\right], n \geq 1$ and $0 \leq a<1$. Let $x_{n}, n \geq 1$ be a sequence of independent random variables with distributions

$$
\begin{array}{rlrl}
P\left(X_{n}=x\right) & =(1-a) / 2 p_{n} & & \text { for } x= \pm \sqrt{p_{n}}, \\
& =a / 2 & & \text { for } x= \pm 1, \\
& =(1-a)\left(1-1 / p_{n}\right) & \text { for } x=0, \text { for each } n \geq 1 .
\end{array}
$$

It is easy to see that $\operatorname{EX}_{n}=0$ and $\operatorname{Ex}_{n}^{2}=1$ for all $n \geq 1$. Let

$$
H(x)=\sup _{n \geq 1} P\left(\left|x_{n}\right| \geq x\right), \quad x \geq 0
$$

We now have that 


$$
\int_{0}^{\infty}\left(x / L_{2} x\right) H(x) d x<\infty
$$

and

$$
(1 / n) \sum_{i=1}^{n} \operatorname{Ex}_{i}^{2} I\left(\left|X_{i}\right| \geq\left(i L_{2} i\right)^{1 / 2}\right)=1 / n+((n-1) / n)(1-a) \rightarrow 1-a
$$

as $n \rightarrow \infty$. Consequently,

$$
\limsup { }_{n \rightarrow \infty} S_{n} / \sqrt{2 n L_{2} n}=a \text { a.s. }
$$

and

$$
\liminf _{n \rightarrow \infty} S_{n} / \sqrt{2 n L_{2} n}=-a \quad a . s .
$$

From Examples 3 and 4 , we know that the conditions in Theorem 2.5 are, in some sense, not only optimal, but also the value of the limit supremum of $s_{n} /\left[s_{n}\left(2 L_{2} s_{n}^{2}\right)^{1 / 2}\right]$ depends on the value of the limit of $s_{n}^{-2} \sum_{i=1}^{n} E\left(x_{i}^{2} I\left(\left|x_{i}\right| \geq\left(i L_{2} i\right)^{1 / 2}\right)\right)$.

The referee has pointed out the following. Theorem A, which plays a crucial role in the establishment of Theorem 2.2 , obtains for more general sequences $a_{n}, n \geq 1$ satisfying only $a_{n} / s_{n} \uparrow \infty$. But Theorem 2.2 was proved for the particular choice $a_{n}=s_{n}\left(L_{2} s_{n}^{2}\right)^{1 / 2}, n \geq 1$ of weights. Can there be a version of Theorem 2.2 for general sequences $a_{n}, n \geq 1$ ?

ACKNOWLEDGMENTS. The authors are delighted to acknowledge the meticulous care the referee has taken in scrutinizing our paper. The referee has brought to our attention the works of Teicher and Tomkins which also deal with the general theme of this paper. A careful review of the papers by Teicher and Tomkins led us to make a critical comparison of our work with their work and thus enhancing the value of our work. Mr. Deli Li gratefully acknowledges the support of Youth Science Foundation of China for his research. Professor Wang gratefully acknowledges the support of the National Natural Science Foundation of China for his research. 
1. BIKELIS, A. Ocenkı ostatočnogo člena v central'no $\check{i}$ predel'no $\bar{i}$ teoreme. Litovski $\check{\text { matem. }}$ sb.,$\underline{6}$ (1966), No.3, 323-346.

2. BUTZER, P.L. and HAHN, L. General Theorems for Rates of Convergence in Distribution of Random Variables, I : General Limit Theorems, Journal of Multivariate Anal., $\underline{8}$ (1978), 181-208.

3. EGOROV, V.A. Obobscenie teoremy Hartmana-Vintnera o zakone povtornogo logarifma, Vestnik Leningrad. Univ., (1971), No.7, 22-28.

4. FELLER, W. On the Oscillations of Sums of Independent Random Variables, Ann. Math. , 91 (1970), No.2, 402-418.

5. FRIEDMAN, N., KATZ, M. and KOOPMANS, L.H. Convergence Rates for the Central Limit Theorem, Proc. Nat.Acad.Sci. USA, 56 (1966), 1062-1065.

6. HARTMAN, P. and WINTNER, A. On the Law of the Iterated Logarithm, Amer. J. Math. , 63 (1941), No.1, 169-176.

7. KATZ, M. Note on the Berry-Esseen Theorem, Ann. Math. Stat., 34 (1963), No.3, 1107-1108.

8. LEDOUX, M. and TALAGRAND, M. Characterization of the Law of the Iterated Logarithm in Banach Spaces, Ann.Probab., $\underline{16}$ (1988), No.3, 1242-1264.

9. PETROV, V.V. Sums of Independent Random Variables, Springer-Verlag, New York, 1975.

10. TEICHER, H. On the Law of the Iterated Logarithm, Ann.Probab., $\underline{2}$ (1974), 714-728.

11. TOMKINS, R.J. Lindeberg Functions and the Law of the Iterated Logarithm, $\underline{Z}$. Wahrscheinlichkeits theorie Verw.Gebiete, $\underline{65}$ (1983), 135-143.

12. TOMKINS, R.J. A Generalized Law of the Iterated Logarithm, Statistics \& Probability Letters, 10 (1990), 9-15.

13. WITTMANN, R. A General Law of Iterated Logarithm, Z. Wahrscheinlichkeitstheorie. Verw. Gebiete, 68 (1985), 521-543.

14. mitTManN, R. Sufficient Moment and Truncated Moment Conditions for the Law of the Iterated Logarithm, Probab. Th. Rel. Fields, $\underline{75}$ (1987), 509-530. 


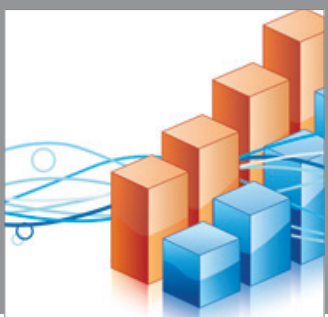

Advances in

Operations Research

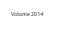

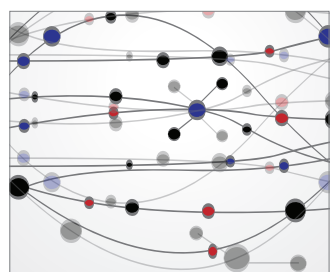

\section{The Scientific} World Journal
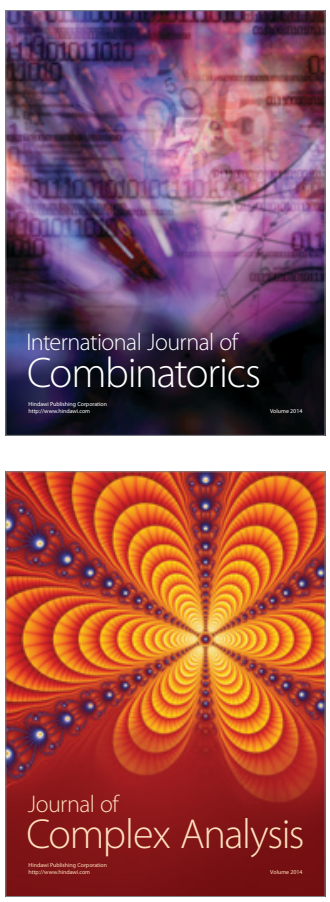

International Journal of

Mathematics and

Mathematical

Sciences
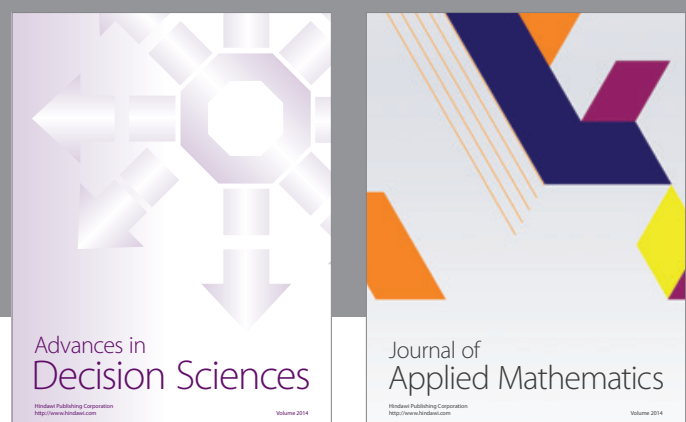

Journal of

Applied Mathematics
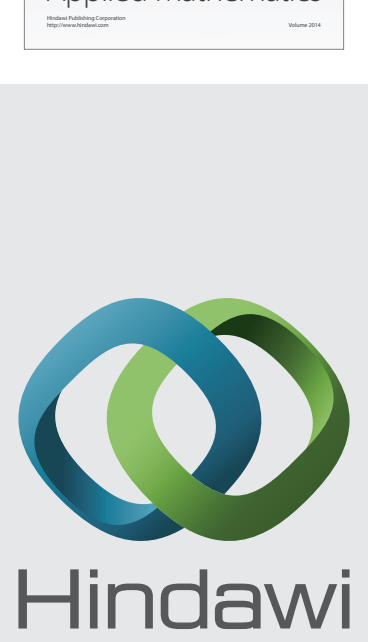

Submit your manuscripts at http://www.hindawi.com
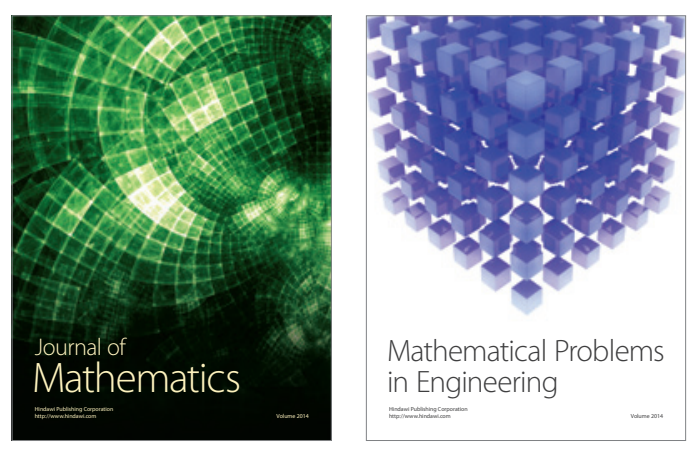

Mathematical Problems in Engineering
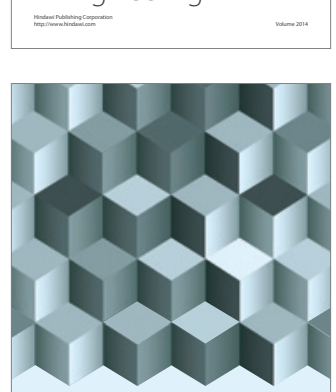

Journal of

Function Spaces
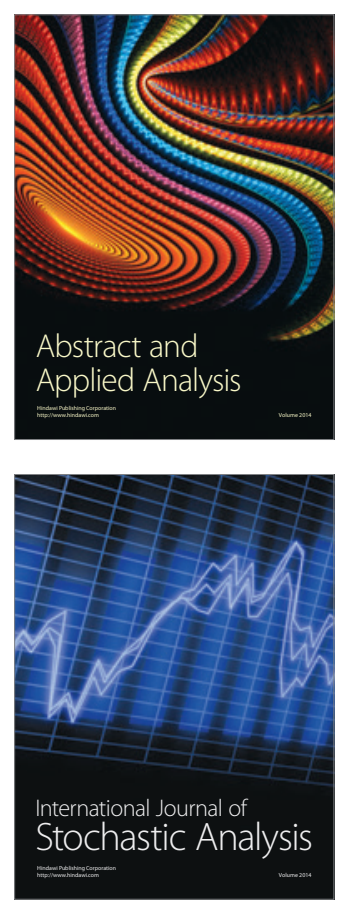

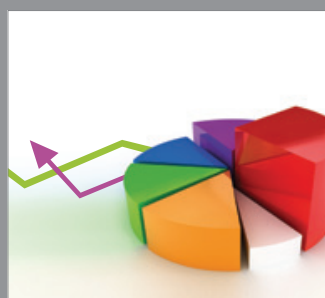

ournal of

Probability and Statistics

Promensencen
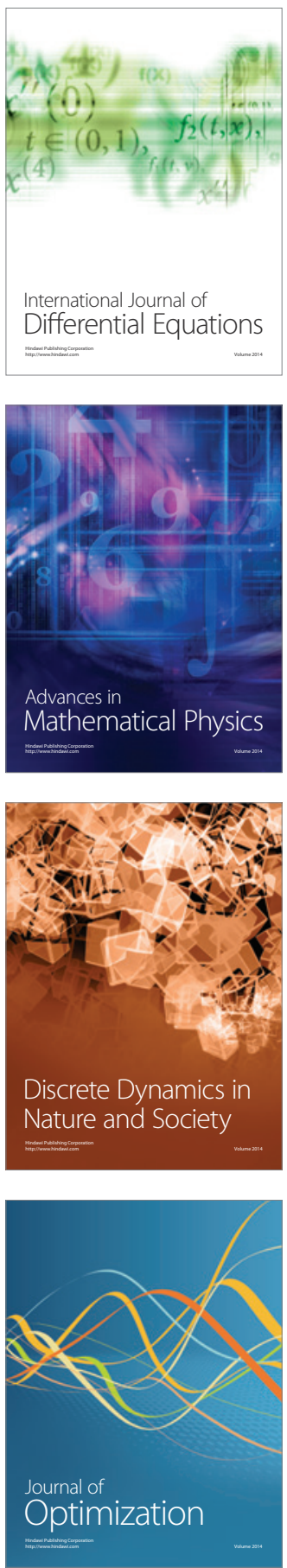